\title{
Discussion: Immunology, cellular pathology, genetics
}

\author{
C Ritchlin
}

Ann Rheum Dis 2005;64(Suppl II):ii40-ii41. doi: 10.1136/ard.2004.031559

\begin{abstract}
What is the evidence suggesting that genetic factors are important in the pathogenesis of psoriatic arthritis (PsA)?
\end{abstract}

Much of the evidence supporting the genetic basis of PsA is derived from twin studies, family based investigations, and population based epidemiologic studies. Concordance rates of PsA in twins are unknown, but monozygotic twins have a threefold increased risk for psoriasis compared with fraternal twins. A population study conducted by Moll and Wright indicated the overall prevalence of PsA among first degree relatives was $5.5 \%$ and that the risk for affected first degree relatives $\left(\lambda_{1}\right)$ is 55 . This compares to a reported $\lambda_{1}$ of 8 for psoriasis. One family study reported that if one sibling had PsA, the corresponding risk for another sibling to develop the disease was $0.10,0.22$, and 0.31 if no parent was affected, the mother was affected, or the father was affected, respectively.

Which genetic factors have been associated with PsA? The commonest approaches to identify specific genetic factors include positional cloning studies that involve collections of families with multiple affected individuals or analysing affected sibling pairs so that linkage analysis can be performed. One genome-wide scan in 178 Icelandic patients reported an LOD score of 2.17 on chromosome 16q, whereas conditional on paternal transmission to affected individuals, the LOD score was 4.19. Recent studies of patients with PsA in Newfoundland suggest that variants within the CARD15 locus on chromosome 16q contribute to disease susceptibility.

Another approach for pinpointing genetic factors includes association studies. The strongest associations in PsA and psoriasis have been noted in the genes of the major histocompatibility complex (MHC) region. In particular, the class I human leucocyte antigens HLA-B13, HLA-B17 and its split HLA-B57, and HLA-Cw6 have demonstrated a positive association across different population studies. Interestingly, individual alleles may be associated with certain features of PsA. For example, HLA-CW ${ }^{*} 0602$ is associated with an earlier age of onset of psoriasis, HLA-B27 with axial involvement, and HLA-B38 and HLA-B39 with peripheral polyarthritis. One of the major challenges of association studies in PsA is that investigators are faced with a "disease within a disease". Thus, the HLA links may be related to the psoriasis and not to the arthritis. Certainly, larger studies will be required to understand better whether genetic factors, which predispose to arthritis and not to the skin disease, can be identified.

\section{Which cytokines have been implicated in the pathogenesis of PsA?}

Studies on psoriatic synovial membranes have revealed a $\mathrm{T}$ helper 1 (Th 1) pattern of cytokine production characterised by elevated levels of interferon $\gamma$ (IFN $\gamma$ ) and interleukin (IL)2. The Th 2 cytokines IL-4 and IL-5 were not identified in psoriatic synovium. This pattern of cytokine production is similar to that reported in psoriasis with one important difference. The anti-inflammatory cytokine IL-10 was released in large quantities by cultured psoriatic synovial explants but not skin explant tissues. Additionally, both tumour necrosis factor $\alpha$ (TNF $\alpha)$ and IL-1 $\beta$ were strongly expressed in psoriatic synovial membranes and explant tissues. Recent studies show that the cytokines IL-15 and IL-18, important in innate immunity, are also expressed in psoriatic synovium.

What is the evidence (pro and con) that CD8 + T cells are important in the pathogenesis of PsA?

The association between PsA and class I MHC alleles provided preliminary evidence that CD8+ T cells may be of central importance in synovial inflammation. In this scheme, an antigen, presumably an infectious agent, triggers an aberrant $\mathrm{CD} 8+\mathrm{T}$ cell response that mediates subsequent downstream inflammatory events. This model has been supported by studies indicating that psoriasis and PsA dramatically worsened in patients with acquired immune deficiency syndrome (AIDS) as the CD4+ cell counts dropped. Additional evidence came from studies of psoriatic synovial fluid showing a predominance of CD8+ T cells, some of which appear to have been generated by an antigen driven mechanism.

CD4+ $\mathrm{T}$ cells are the predominant subset in psoriatic synovial membranes with ratios of CD4 to CD8 cells as high as $4: 1$. Secondly, recent studies from Zambia indicate that PsA improves with the onset of AIDS in human immunodeficiency virus (HIV) positive patients. Lastly, a dominant role for $\mathrm{T}$ cells in PsA has been challenged by a recent randomised study showing that efalizumab, an agent that inhibits $\mathrm{T}$ cells, significantly lessened psoriasis activity but not joint inflammation in patients with PsA.

What is the vascular morphology of the psoriatic plaque and synovial membrane, and which cytokines may be driving the vascular remodelling?

Arthroscopic studies have demonstrated that PsA synovial membranes display a highly tortuous morphology compared with vessels in rheumatoid joints, which are more linear in appearance. This tortuous pattern has also been noted in psoriatic skin lesions. Moreover, several studies have noted that psoriatic synovial membranes are more vascular than rheumatoid arthritis (RA) tissues. Immunohistochemical studies on psoriatic synovial membranes indicate that vascular endothelial growth factor (VEGF) and angiopoietin-2 may play an important role in the development of the tortuous morphology. In particular, angiopoietin-2 is thought to regulate a more disordered pattern of new blood vessel development. These molecules were more highly expressed in PsA compared with RA synovium and the expression of both molecules decreased after treatment with methotrexate. The significance of vascular tortuosity in disease pathogenesis is obscure but conceivably altered vascular remodelling could

Abbreviations: DC, dendritic cell; HLA, human leucocyte antigen; IL, interleukin; MHC, major histocompatibility complex; PsA, psoriatic arthritis; RA, rheumatoid arthritis; RANKL, receptor activator of nuclear factor $\kappa B$ ligand; TNF, tumour necrosis factor 
enhance inflammation by facilitating entry of inflammatory cells from the circulation into the joint.

Which subsets of dendritic cell have been identified in the psoriatic plaque, and how do these cells contribute to the cutaneous inflammation?

Dendritic cells (DCs) are present in large quantities in psoriatic skin lesions and several different subtypes have been identified. Langerhans cells-resident immature DCs in normal skin-are often increased in psoriatic plaques. A second type of immature DC population, derived from circulating CDl4+ monocytes, is also greatly expanded in psoriatic skin. These cells express MHC class II molecules and $\mathrm{CD} 1 \mathrm{lc}$ and develop into mature DCs in response to IFN $\alpha$. The mature monocytoid DCs are major producers of IL-12 and IL23 polarising the $\mathrm{T}$ lymphocyte response towards a Th $\mathrm{l}$ profile. The mature monocytoid DCs can also activate T cells by presenting antigen. A third DC subset, termed the plasmacytoid DC, is also greatly increased in psoriatic skin. These cells can produce large quantities of IFN $\alpha$ tipping the balance towards monocytic DC maturation and $\mathrm{T}$ cell activation.

\section{What are the key cellular and molecular events that} mediate bone resorption in the psoriatic joint?

Large eccentric bone erosions, extensive tuft resorption, and pencil in cup deformities coupled with bony ankylosis indicate the presence of dysregulated bone remodelling in psoriatic joints. The mechanisms underlying new bone formation are not well understood, but excessive bone resorption or osteolysis is mediated by osteoclasts. Increased numbers of osteoclasts have been identified at the bone-pannus junction in psoriatic joints. Also, the receptor activator of nuclear factor $\kappa \mathrm{B}$ ligand (RANKL), a molecule that promotes osteoclastogenesis, has been shown to be upregulated in the psoriatic synovial lining layer while osteoprotegerin, a molecule that inhibits osteoclastogenesis, was present in low levels in perivascular structures. Additional studies have revealed the presence of increased numbers of osteoclast precursors in the peripheral blood of patients with PsA compared with healthy controls. These cells, derived from CDI4+ monocytes, presumably enter the synovial membrane through blood vessels and differentiate into osteoclasts after exposure to relatively unopposed RANKL. In studies in transgenic TNF mice and patients with PsA the frequency of circulating osteoclast precursors was increased by TNF $\alpha$.

What is the evidence supporting the concept that TNF is a critical cytokine mediating inflammatory events in the psoriatic joint?

At least four lines of evidence support the concept that TNF is a pivotal cytokine in PsA. Firstly, TNF levels are elevated in the peripheral blood and synovial fluid of patients with PsA. Secondly, TNF release is upregulated in synovial explant tissues and increased expression of TNF protein has been observed in synovial membranes stained with anti-TNF antibodies. TNF is largely confined to cells located in the lining layer. Thirdly, clinical trials with anti-TNF agents have demonstrated marked clinical efficacy, and these agents dramatically slowed radiographic progression. Biopsy of synovial tissues before and after therapy with anti-TNF agents showed a significant decline in inflammation as early as two weeks after therapy. Fourthly, anti-TNF agents rapidly decrease the level of circulating osteoclast precursors and reduce bone marrow oedema on fat suppressed magnetic resonance imaging.

Correspondence to: $\operatorname{Dr} C$ Ritchlin, Clinical Immunology Research Center, University of Rochester Medical School, Rochester, NY, USA; christopher_ritchlin@urme.rochester.edu 